\title{
Fetomaternal outcome in patients with oligohydromnions in a tertiary hospital
}

\section{Ratnaboli Bhattacharya, Akshaya S.*}

Department of Obstetrics and Gynaecology, Kasturba Hospital, Delhi, India

Received: 24 June 2016

Accepted: 29 June 2016

\section{*Correspondence:}

Dr. Akshaya S.,

E-mail: akshayadr2010@gmail.com

Copyright: (c) the author(s), publisher and licensee Medip Academy. This is an open-access article distributed under the terms of the Creative Commons Attribution Non-Commercial License, which permits unrestricted non-commercial use, distribution, and reproduction in any medium, provided the original work is properly cited.

\section{ABSTRACT}

Background: Oligohydramnios defined as an AFI $\leq 5$ or maximum vertical pocket devoid of umbilical cord or fetal limbs measure $<2 \mathrm{~cm}$, in the presence of intact membranes is a common obstetric complication. The objective was to study the fetomaternal outcome in patients with oligohydromnios in tertiary hospital.

Methods: 50 singleton live pregnancies between 34-40 weeks gestation with well-established dates with AFI 5 or less were selected for the study group. 50 singleton live pregnancies between 34-40 weeks gestation with well-established dates with AFI more than 5 were selected for the control group. Ultrasound examination was done for all women whenever they visited the hospital at/after 34 weeks of gestation. AFI was obtained sonographically. Maternal and fetal outcomes were studied in both groups.

Results: Oligohydromnios is associated with increased rates of LSCS and induction of labour. The babies are more likely for NICU admissions. There was no difference in low APGAR score, congenital anamoly, perinatal deaths.

Conclusions: The study concludes that oligohydromnios leads to increased LSCS, induction and NICU admissions. Once oligohydramnios is detected, patients should be put on regular fetal surveillance such as Doppler studies and NST. Timely termination of pregnancy can reduce the fetal and maternal complications and improve labour outcome.

Keywords: Oligohydromnios, AFI, Induction, LSCS, Perinatal morbidity

\section{INTRODUCTION}

Amniotic fluid is necessary for fetal growth and development. It protects the fetus from trauma, infection and cushions the umbilical cord from compression between the fetus and the uterus. It provides necessary fluid and growth factors and maintains an even temperature. ${ }^{1}$ Oligohydramnios defined as an AFI $\leq 5$ or maximum vertical pocket devoid of umbilical cord or fetal limbs measure $<2 \mathrm{~cm}$, in the presence of intact membranes is a common obstetric complication occurring in $3-5 \%$ pregnancies at term. ${ }^{1,2}$ In general, oligohydramnios developing early in pregnancy is less common and frequently has a bad prognosis. ${ }^{3}$ The common maternal conditions associated with oligohydramnios are ruptured membranes, postmaturity, hypertension, autoimmune disorders and maternal medications like prostaglandin synthetase inhibitors. Fetal factors are essentially related to placental insufficiency, intrauterine growth restriction and congenital anomalies, particularly of the renal tract. ${ }^{3}$

Some authors think that decreased AFV is a predictor of poor fetal tolerance of labour and is associated with increased risk in abnormal heart rate, caesarean section and meconium stained amniotic fluid. There is also increased risk of Apgar score $<7$ in these cases. ${ }^{4-6}$ Oligohydramnios is associated with fetal genitourinary anomalies, postural deformities and increased risk of perinatal morbidity.

Sequelae of prolonged oligohydramnios includes fetal compression syndrome and pulmonary hypoplasia due to compression of the fetal abdomen which limits the 
movement of the fetal diaphragm and decreases the flow of amniotic fluid into and out of the fetal lung. ${ }^{7}$

However, over the last decades, some authors have found that diminished amniotic fluid index alone is not associated with intrapartum complications, increased caesarean rates or other adverse perinatal outcomes. ${ }^{8-10}$

The intention of this study was to find out whether an AFI of $5 \mathrm{~cm}$ or less is related to any fetal or maternal factors and the effect it has on the progress of labour and the mode of delivery along with the perinatal outcome.

\section{METHODS}

A prospective cohort study was conducted in the department of obstetrics and gynaecology, Kasturba hospital, Delhi, India from March 2013 to March 2014. The study was conducted after ethical clearance from the institutional ethical committee.

50 singleton live pregnancies between 34-40 weeks gestation with well established dates with AFI 5 or less were selected for the study group. 50 singleton live pregnancies between 34-40 weeks gestation with well established dates with AFI more than 5 were selected for the control group. Those who remembered the date of their last menstrual period correctly with previous regular cycles and/or had corresponding first trimester ultrasound were taken for the study. Patients with multiple pregnancies, with IUD at the time of diagnosis or with previous history of uterine surgeries which preclude trial of labour were excluded from the study. Apart from a detailed history and examination, ultrasound examination was done for all women whenever they visited the hospital at/after 34 weeks of gestation. Fetal maturity, biometry and morphology scan, fetal presentation and placental localization was done. AFI was obtained sonographically. If the AFI was less than or equal to $5 \mathrm{~cm}$, the four quadrant evaluation was performed three times and the values were averaged.

All the women underwent Doppler studies and biophysical scoring. Those with deranged values were repeated weekly and those with normal values were repeated every 15 days until pregnancy termination. Parameters studied were S/D ratio and RI and PI offetal umbilical artery and middle cerebral artery. Biophysical scoring included the following parameters-fetal breathing movements, fetal movements, fetal tone, amniotic fluid and NST. NST tracings were taken for all the women included in the study. Patients with abnormal Doppler studies and biophysical scoring had NST done on alternate days while those with normal values were done weekly till pregnancy was terminated. Patients in study group and control group who had normal Doppler and biophysical values were followed up with Doppler studies every 15 days and weekly NST. Patients of study group who had no other associated medical or obstetric disorders were allowed to go into labour spontaneously till 37 weeks, after which they were terminated. Such patients in control group were left for spontaneous onset of labour till 40 weeks after which pregnancy was terminated. Labour was augmented with oxytocin in those who went into labour spontaneously or induced with dinoprostone gel along with oxytocin augmentation depending on the Bishop's score of the patients or taken up for caesarean section if indicated.

Patients in both groups who had normal Doppler and NST studies but had other medical or obstetric complications like eclampsia, pre-eclampsia, GDM, IUGR etc. were terminated as and when indicated. These patients were followed up till termination with Doppler studies weekly and NST once weekly. The mode of termination was either induction with dinoprostone gel or caesarean section, whichever was indicated. Patients in both groups who had deranged Doppler values had Doppler studies repeated weekly and NST on alternate days. Abnormal Doppler studies like absent diastolic flow or reversed diastolic flow or abnormal fetal heart tracings were either induced with dinoprostone gel or taken up for caesarean section as and when indicated. Those who developed variable deceleration and late deceleration with or without meconiurn stained liquor which persisted after corrective measures like maternal position, oxygen inhalation, hydration and stoppage of oxytocin were taken up for LSCS.

Patients with cephalopelvic disproportion, malpresentations like breech, transverse lie or unstable lie, major degrees of placenta previa were taken up for elective caesarean section.

Patients for emergency caesarean section were those with dystocia, fetal distress, failed trial of labour, failed induction of labour, antepartum haemorrhage, abruption, cord prolapsed or fulminant pre-eclampsia/eclampsia with unfavourable cervix.

All cases were monitored by continuous electronic fetal monitoring during labour and followed till delivery, the gestational age at delivery was seen and labour monitoring along with active management of labour was done. Whether labour was induced, augmented or spontaneous in onset, the progress of labour, duration of labour and whether delivery took place by normal vaginal route, by instrumentation or by caesarean section was observed. The number of patients who successfully withstood the process of labour and those who had failed instances of induction or development of intrapartum complications was noted. The number and indications for patients taken up for both elective caesarean sections and emergency caesarean sections was documented. The nature of the amniotic fluid was noted at spontaneous or artificial rupture of membranes during labour or caesarean section.

All the new borns were attended by pediatricians and perinatal variables were determined by them. Various 
outcome measures recorded were Apgar score at 1 minute and 5 minutes, birth weight, IUGR, admission to NICU, congenital anomalies, perinatal morbidity and mortality. Neonates were followed up for 7 days.

\section{Statistical analysis}

Data was entered in a database file on SPSS 14 and analysed using the same software. Data was compared using chi-square or Fischer's test where appropriate and continuous variables were analyzed with student's t-test. A p-value of $<0.05$ or an odds ratio (OR) with $95 \%$ confidence interval (CI) not inclusive of the unity was considered significant.

\section{RESULTS}

The patients were thus divided into two groups according to $\mathrm{AFI}$;

Study group: patients with AFI $\leq 5$; control group: patients with AFI $>5$.

Table 1 shows the distribution of patients according to maternal variables. The two groups were comparable on the basis of age and gravidity. However the difference in booking status was significant in the two groups. The mode of termination has been described in Table 2 .

Table 1: Maternal variables in the study.

\begin{tabular}{|lll|}
\hline & $\begin{array}{l}\text { Study group } \\
(\mathrm{AFI} \leq 5)\end{array}$ & $\begin{array}{l}\text { Control group } \\
(\mathrm{AFI}>5)\end{array}$ \\
\hline Mean age (years) & $25.21 \pm 3.166$ & $25.25 \pm 2.911$ \\
\hline Booked Patients & $64 \%^{*}$ & $78 \%^{*}$ \\
\hline Mean gravidity & $2.20 \pm 1.044$ & $2.14 \pm 1.045$ \\
\hline Mean AFI & $3.605 \pm 1.1490 \mathrm{~cm}$ & $12.001 \pm 3.520 \mathrm{~cm}$ \\
\hline${ }^{*} \mathrm{p} \leq 0.05$ & & \\
\hline
\end{tabular}

Table 2: Mode of pregnancy termination in the study.

\begin{tabular}{|lll|}
\hline NVD & $\begin{array}{l}\text { Study group } \\
(\text { AFI } 5 \text { 5) }\end{array}$ & $\begin{array}{l}\text { Control group } \\
(\text { AFI }>5)\end{array}$ \\
\hline Elective LSCS & 40 & 64 \\
\hline Induction of labour & 9 & 11 \\
\hline $\begin{array}{l}\text { Emergency LSCS for } \\
\text { fetal distress }\end{array}$ & 15 & 30 \\
\hline $\begin{array}{l}\text { Emergency LSCS for } \\
\text { other reasons }\end{array}$ & 9 \\
\hline $\begin{array}{l}\text { Instrumental vaginal } \\
\text { delivery }\end{array}$ & 3 & 5 \\
\hline
\end{tabular}

In the study group (AFI $\leq 5), 23(40 \%)$ patients were induced because they had oligohydramnios and abnormal Doppler, $13(23 \%)$ patients because they had abnormal Doppler and other medical/obstetric complications like pre-eclampsia and GDM. $8(14 \%)$ patients were induced just because of their associated medical/obstetric problems like hypertension and GDM. 11 (19\%) patients were induced because they had oligohydramnios and gestational age was either equal to or $>37$ weeks. $1(2 \%)$ patient was induced because she had oligohydramnios and MSL. In the control group (AFI >5), 8 (27\%) patients were induced because they had abnormal Doppler. $8(27 \%)$ patients were induced because they had abnormal Doppler associated with other medical/obstetric complications like pre-eclampsia and GDM, 1 (3\%) patient because of MSL. $13(43 \%)$ patients were induced because they had completed 40 weeks of gestation. The mode of delivery between the two groups was statistically significant. However the number of instrumentations and differences in indications for Em.LSCS was statistically insignificant. The mean gestational age at delivery of the study group was $36.26 \pm 0.991$ standard deviation weeks and control group was $37.90 \pm 1.307$ standard deviation weeks $(\mathrm{p}=0.00)$. Table 3 depicts the neonatal outcome of the patients. In the study group, $57 \%$ patients had baby weights less than $2.0 \mathrm{~kg}$. In $16 \%$ patients birth weights were between $2.0-2.9 \mathrm{~kg}$, in $23 \%$ between $3.0-3.9 \mathrm{~kg}$ and in $4 \%$ patients baby weight was $\geq 4 \mathrm{kgs}$. In the control group, $24 \%$ patients had birth weights less than $2.0 \mathrm{~kg}$. $42 \%$ patients had baby weights between $2.0-2.9 \mathrm{~kg}, 30 \%$ patients between $3.0-3.9 \mathrm{~kg}$ and in $4 \%$ patients baby weight was $\geq 4 \mathrm{kgs}$. The mean birth weight of the study group was $2.37 \pm 0.77 \mathrm{~kg}$ and of the control group was $2.70 \pm 0.68 \mathrm{~kg}$. The difference was statistically significant.

Table 3: Fetal outcomes in the study.

\begin{tabular}{|lll|}
\hline $\begin{array}{l}\text { Neonatal outcome } \\
\text { Low Apgar scores } \\
\text { (5 min) }\end{array}$ & $\begin{array}{l}\text { Study group } \\
(\mathrm{A} F \mid \leq 5)(\%)\end{array}$ & $\begin{array}{l}\text { Control group } \\
(\mathrm{AFI}>5)(\%)\end{array}$ \\
\hline NICU admissions & 11 & 5 \\
\hline Perinatal deaths & $65^{*}$ & $28^{*}$ \\
\hline Congenital anomalies & 7 & 3 \\
\hline${ }^{*} \leq 0.05$ & & 3 \\
\hline
\end{tabular}

\section{DISCUSSION}

The evaluation of the Amniotic Fluid Volume has become an integral component of the fetoplacental assessment and surveillance of pregnancies that are considered to be at risk for an adverse pregnancy outcome. ${ }^{11}$ In this study, maximum number of cases were found in the age group of 21-30 years for both the groups $89(89 \%)$ patients in the study group (AFI=5) and 92 $(92 \%)$ patients in the control group (AFI $>5)$. The mean age for study group was 25.21 33.166 years standard deviation while the mean age for control group was $25.25 \pm 2.911$ years standard deviation. The two groups were thus found to be comparable on the basis of age $(\mathrm{p}=0.6)$.

Bangal et al found that the mean $( \pm \mathrm{SD})$ maternal age for patients with oligohydramnios was $22.8 \pm 4.2$ years. ${ }^{12}$ The results of present study were similar to that found by 
Guin et al. where the mean age for patients with oligohydramnios was $24.71 \pm 4.58$ years. ${ }^{6}$ In this study, 36 (36\%) patients with $\mathrm{AFI}=5$ were unbooked, only 22 (22\%) patients with AFI $>5$ were unbooked. This difference was found to be statistically significant $(p=0.029)$. This was because unbooked patients do not receive routine antenatal care which increases the number of pregnancy complications in them thereby leading to fetal compromise and oligohydramnios.

Booking status has not been compared in other studies. In this study, $31(31 \%)$ patients in the study group (AFI=5) were primigravida and $69(69 \%)$ patients were multigravidas. $32(32 \%)$ patients in the control group $(\mathrm{AFI}>5)$ were primigravida and the rest $68(68 \%)$ were multigravidas. The two groups were comparable on the basis of gravidity ( $\mathrm{p}=0.874)$. These figures differed from that found by Jandial et al. where $68 \%$ patients were primigravidas. ${ }^{13}$

The mean AFI in the study group (AFI=5) was $3.605 \pm 1.1490 \mathrm{~cm}$. These figures matched with those found by Driggers et al where the mean AFI was $3.0 \pm 1.5 \mathrm{~cm} .{ }^{14}$ In this study, the mean AFI of the control group (AFI >5) was $12.001 \pm 3.520 \mathrm{~cm}$. This was slightly more than that found by Driggers et al. $10.7 \pm 4.0 \mathrm{~cm} .{ }^{14}$

In the study group (AFI=5), $57(57 \%)$ patients had to be induced as compared to $30(30 \%)$ patients in the control group (AFI $>5$ ). Elective LSCS was done on $9(9 \%)$ patients in the study group and $11(11 \%)$ patients in the control group. 34 (34\%) patients went into spontaneous labour in the study group and $59(59 \%)$ patients in the control group. This difference was statistically significant $(p=0.00)$ The number of inductions were more in patients with oligohydramnios because of abnormal Doppler and/or medical/obstetric complications (like eclampsia, pre-eclampsia, GDM) and because pregnancies were not allowed to continue beyond 37 weeks in these patients. Comparative rate of elective LSCS done for AFI=5 and AFI $>5$ are similar. Ahmad et al showed that $63.4 \%$ of patients with $\mathrm{AFI}=5$ were induced in comparison to $14 \%$ patients with AFI $>5$ which agreed with this study. ${ }^{15}$ Sultana et al. found that more inductions of labour were done in low AFI than normal AFI women and this was statistically significant $(\mathrm{p}=0.04) .{ }^{16}$

Indications for induction of labour in this study matched with those found by Ahmad et al. who deduced that $63.4 \%$ patients with oligohydramnios had to be induced for foetal cause. On the other hand, $14 \%$ of patients with normal AFI were induced and they were all due to maternal medical/ obstetric problems. ${ }^{15}$ This research found that in the study group (AFI=5), 3 (3\%) patients had instrumental vaginal delivery, 57 (57\%) patients underwent LSCS- $9(9 \%)$ patients were decided on elective basis and $48(48 \%)$ patients had to be taken up as emergency. In the control group (AFI >5), $5(5 \%)$ patients had instrumental vaginal delivery, only $31(31 \%)$ patients underwent LSCS- $11(11 \%)$ patients were taken up electively and 20 (20\%) patients were emergency LSCS. This difference was statistically significant $(p=0.00)$. The number of emergency LSCS was more in the study group due to the increased number of patients who had fetal distress and non-progress of labour. Cord compression, head compression and uteroplacental insufficiency are important contributors to this. Increased incidence of LSCS for fetal distress was found in our study due to usage of advanced intrapartum monitoring devices Chate et al. showed that $64 \%$ oligohydramnios patients had to undergo LSCS in comparison to $22 \%$ with adequate AFI. ${ }^{17}$ Guin et al concluded that overall $42.8 \%$ oligohydramnios patients underwent LSCS. ${ }^{6}$ This figures agreed with these 2 studies. $69 \%$ of study group and $55 \%$ of control group had emergency LSCS due to fetal distress. Jandial et al found that $42 \%$ caesarean sections were due to fetal distress in patients having oligohydramnios. ${ }^{13}$ It was found that $80 \%$ of the caesarean sections were due to fetal distress in patients with low AFI in a study done by Guin et al. ${ }^{6}$ Currently most obstetricians faced with an AFI of less than $5 \mathrm{~cm}$ admit the patient and induce labour if pregnancy is at or more than 37 completed weeks of gestation. This induction of labour is accompanied by increased caesarean rate.

The low Apgar score at $5 \mathrm{~min}$ was found in $11 \%$ of the study group and $5 \%$ of the control group. This difference was not statistically significant. Sultana et al. also did not find any statistically significant difference in the Apgar scores between two groups. ${ }^{9}$ In this study, 65 (65\%) babies in the study group (AFI=5) were admitted in NICU as compared to $28(28 \%)$ babies in the control group (AFI >5). This difference was statistically significant in this study $\mathrm{p}=0.00$. Umber et al. showed that $7 \%$ of babies with oligohydramnios were taken to the NICU in comparison to $1.7 \%$ patients with adequate AFI. ${ }^{4}$ The number of NICU admissions in this study is more than other studies because all low birth weight babies $(<2.0 \mathrm{~kg})$, pre-term babies ( $<36$ weeks completed) and babies with birth asphyxia were admitted to NICU. Gross congenital anomalies were present in $2(2 \%)$ patients in the study group (AFI=5) and $3(3 \%)$ patients in the control group (AFI >5). This difference was not statistically significant. It is less than that of study by Guin et al $(8.5 \%){ }^{6}$ The perinatal deaths is study group was $7 \%$ and control group was $3 \%$. Guin et al found $2.9 \%$ perinatal mortality in patients having oligohydramnios, similar to that found in this study. ${ }^{6}$

\section{CONCLUSION}

Oligohydromnios is an high risk pregnancy. It led to increased risk of induction of labour and increased LSCS. The babies are more likely for NICU admissions. There was no difference in low Apgar score, congenital anamoly, perinatal deaths. The results of this study, indicate that oligohydramnios $(\mathrm{AFI}=5)$ is associated with increased perinatal morbidity, which is modified by intervention. Stress should be placed on regular antenatal 
check-ups as unbooked patients are more likely to have oligohydramnios and other associated problems. Once oligohydramnios is detected, patients should be put on regular fetal surveillance such as Doppler studies and NST. Timely termination of pregnancy can reduce the fetal and maternal complications and improve labour outcome.

Funding: No funding sources

Conflict of interest: None declared

Ethical approval: The study was approved by the Institutional Ethics Committee

\section{REFERENCES}

1. Dutta DC. The placenta and the membranes. Textbook of obstetrics. $6^{\text {th }}$ edition. Central Book Agency (P) Ltd;2004:37-9.

2. Zhang J, Troendle J, Meikle S, Klebanoff MA, Rayburn WF. Isolated oligohydramnios is not associated with adverse perinatal outcomes. $\mathrm{Br} \mathrm{J}$ Obstet Gynaecol. 2004;111(3):220-5.

3. Misra R. Hydramnios and oligohydramnios. In Renu Misra. Ian Donald's Practical Obstetric Problems. $6^{\text {th }}$ edition. BI Publications Pvt. Ltd;2007:364-75.

4. Umber A. Perinatal outcome in pregnancies complicated by isolated oligohydramnios at term. Annals. 2009;15:35-7.

5. Magann EF, Doherty DA, Lutgendorf MA. Peripartum outcomes of high risk pregnancies complicated by oligohydramnios. J Obstet Gynaecol Res. 2010;36(2):268-77.

6. Guin G, Punekar S, Lele P, Khare S. A prospective clinical study of feto-maternal outcome in pregnancies with abnormal liquor volume. J Obstet Gynaecol India. 2011;61(6):652-5.

7. Belman AB, King LR, Kramer SA. Anomalies of the kidney. Clinical Pediatric Urology. $4^{\text {th }}$ edition. Martin Dunitz Ltd;2004:541.

8. Chauhan SP, Washburne JF, Magann EF. A randomized study to assess the efficacy of the amniotic fluid index as a fetal admission test. Obstet Gynecol. 1995;86:9.
9. Sultana S, Khan MAK, Akhtar KAK, Aslam M. Low amniotic fluid index in high risk pregnancy and poor Apgar score at birth. J College Physicians Surgeons Pakistan. 2008;18(10):630-4.

10. Ahmad H, Munim S. Isolated Oligohydramnios is not an indicator for adverse perinatal outcome. J Pak Med Asso. 2009;59:691.

11. Magann EF. Chauhan SP. Doherty DA. Barrillcaux PS, Martin JN, Morrison JC. Predictability of intrapartum and neonatal outcomes with the amniotic fluid volume distribution: a reassessment using the amniotic fluid index, single deepest pocket, and a dye -determined amniotic fluid volume. Am J Obstet Gynecol. 2003;188:1523-8.

12. Bangal VB, Purushottam AG, Bhushan MS. Incidence of oligohydramnios during pregnancy and its effects on maternal and perinatal outcome. J Pharma Biomed Sci. 2011;12(12):5.

13. Jandial C, Gupta S, Sharma S, Gupta M. Perinatal outcome after antepartum diagnosis of oligohydramnios at or beyond 34 weeks of gestation. JK Science. 2007;9(4):213-4.

14. Driggers RW, Holcroft CJ, Blakemore KJ, Graham EM. An $\mathrm{AFI}=5 \mathrm{~cm}$ within 7 days of delivery in the third trimester is nogt associated with decreasing umbilical arterial $\mathrm{pH}$ and base excess. J Perinatology. 2004;24:72-6.

15. Ahmad H, Munim S. Isolated oligohydramnios is not an indicator for adverse perinatal outcome. J Pakistan Med Asso. 2009;59:691.

16. Sultana S, Khan MAK, Akhtar KAK, Aslam M. Low amniotic fluid index in high risk pregnancy and poor Apgar score at birth. J College Physicians Surgeons Pakistan. 2008;18(10):630-4.

17. Chate $P$, Khatri M, Hariharan C. Pregnancy outcome after diagnosis of oligohydramnios at term. Int $\mathbf{J}$ Reprod Contracept Obstet Gynecol. 2013;2(1):23-6.

Cite this article as: Bhattacharya $\mathrm{R}$, Akshaya $\mathrm{S}$.

Fetomaternal outcome in patients with

oligohydromnions in a tertiary hospital. Int $\mathbf{J}$

Reprod Contracept Obstet Gynecol 2016;5:2576-80. 